ANNALES

POLONICI MATHEMATICI

$81.2(2003)$

\title{
Hilbert spaces of analytic functions of infinitely many variables
}

\author{
by O. V. Lopushansky (Kraków) and A. V. Zagorodnyuk (Lviv)
}

\begin{abstract}
We study spaces of analytic functions generated by homogeneous polynomials from the dual space to the symmetric Hilbertian tensor product of a Hilbert space. In particular, we introduce an analogue of the classical Hardy space $H^{2}$ on the Hilbert unit ball and investigate spectral decomposition of unitary operators on this space. Also we prove a Wiener-type theorem for an algebra of analytic functions on the Hilbert unit ball.
\end{abstract}

Introduction. An increasing interest in infinite-dimensional holomorphy in recent years has generated lots of questions concerning subspaces of analytic functions on Banach spaces. In particular, it is of interest to consider analogs of classical function spaces on the unit ball. In a large number of papers (see e.g. [4], [1], [2]) various analogs of the disk algebra or the $H^{\infty}$ algebra for the unit ball of a Banach space are investigated.

The purpose of this paper is to construct and investigate an analogue of the Hardy space $H^{2}$ of analytic functions on the unit ball of an infinitedimensional separable Hilbert space. The idea of the construction comes from the fact that the dual space to the symmetric Banach space $n$-tensor product endowed with a cross-norm is isometric to a subspace of $n$-homogeneous polynomials on the Banach space. Considering a special cross-norm, the so-called Hilbertian cross-norm, we can obtain a natural Hilbert structure on the tensor product of Hilbert spaces. After symmetrization of the tensor product we obtain a predual to a Hilbert space of continuous polynomials.

In Section 1 we study some properties of spaces of homogeneous Hilbertian polynomials. In particular, it is shown that the set of norm attaining polynomials is not dense in the space of Hilbertian polynomials.

In Section 2 we introduce the Hardy-type class $H^{2}(\mathcal{B})$ of analytic functions on the Hilbert unit ball $\mathcal{B}$ as the dual space to the $\ell_{2}$-sum of symmetric

2000 Mathematics Subject Classification: Primary 46G50; Secondary 46G20, 46G25.

Key words and phrases: Hardy classes on Hilbert ball, holomorphic functions on Hilbert ball. 
Hilbertian tensor products. It is again a Hilbert space, and we investigate spectral decomposition of unitary operators on it. Note that the sum of the symmetric Hilbertian tensor products plays an important role in quantum mechanics, where it is called the symmetric Fock space [11].

In Section 3 we consider a function algebra on $\mathcal{B}$ generated by Hilbertian polynomials and investigate its spectrum.

For background on analytic functions on Banach spaces we refer the reader to [7], [8].

1. Hilbertian tensor product and Hilbertian polynomials. Let $E$ be a separable Hilbert space with an orthonormal basis $\left(e_{i}\right)$ and inner product $(\cdot \mid \cdot)$. Denote by $\mathcal{B}:=\left\{x \in E:\|x\|=(x \mid x)^{1 / 2}<1\right\}$ the open unit ball in $E$, and by $\mathcal{T}:=\{x \in E:\|x\|=1\}$ the unit sphere in $E$.

It is known (see e.g. [9, p. 27], [5, p. 351] or [11]) that it is possible to define a norm $h$ on the algebraic tensor product $\otimes^{n} E$ of $E$ such that the completion $\bigotimes_{h}^{n} E$ of $\bigotimes^{n} E$ under this norm is a Hilbert space. More exactly, each vector $w \in \bigotimes_{h}^{n} E$ can be represented as

$$
w=\sum_{i=1}^{\infty} \alpha_{i} e_{1 i} \otimes \ldots \otimes e_{n i},
$$

and we put

$$
\|w\|=\left(\sum_{i=1}^{\infty} \alpha_{i}^{2}\right)^{1 / 2},
$$

where the $e_{k i}$ belong to the orthonormal basis of $E$ and $\alpha_{i} \in \mathbb{C}$. An inner product $(u \mid v)_{n}$ on $\bigotimes_{h}^{n} E$ can be defined by

$$
(u \mid v)_{n}=\sum_{i, j} \alpha_{i} \gamma_{j}\left(e_{1 i} \mid e_{1 j}\right) \ldots\left(e_{n i} \mid e_{n j}\right), \quad \alpha_{i}, \gamma_{j} \in \mathbb{C},
$$

where $u=\sum_{i} \alpha_{i} e_{1 i} \otimes \ldots \otimes e_{n i}, v=\sum_{j} \gamma_{j} e_{1 j} \otimes \ldots \otimes e_{n j} \in \bigotimes_{h}^{n} E$.

Let $x_{i_{1}} \ldots x_{i_{n}}:=(1 / n !) \sum_{\sigma \in \mathcal{G}_{n}} x_{\sigma\left(i_{1}\right)} \otimes \ldots \otimes x_{\sigma\left(i_{n}\right)}$ be the symmetric tensor product of $x_{i_{1}}, \ldots, x_{i_{n}}$ and $x^{n}:=x \otimes \ldots \otimes x$, where $\mathcal{G}_{n}$ is the permutation group of the set $\{1, \ldots, n\}$.

Proposition 1.1. (a) There exists a unique continuous orthogonal projection $S_{n}$ on $\bigotimes_{h}^{n} E$ such that

$$
S_{n}\left(e_{1 i} \otimes \ldots \otimes e_{n i}\right)=\frac{1}{n !} \sum_{\sigma \in \mathcal{G}_{n}} e_{\sigma(1) i} \otimes \ldots \otimes e_{\sigma(n) i} .
$$

(b) The space $E_{h}^{n \prime}$ of continuous linear functionals on $E_{h}^{n}:=S_{n}\left(\bigotimes_{h}^{n} E\right)$ is isometrically isomorphic to some subspace $\mathcal{P}_{h}\left({ }^{n} E\right)$ of the space $\mathcal{P}\left({ }^{n} E\right)$ of $n$-homogeneous continuous polynomials on $E$. 
(c) The image $E_{h}^{n}=S_{n}\left(\bigotimes_{h}^{n} E\right)$ is the closure of the linear span of $x^{n}$ in $\bigotimes_{h}^{n} E$ for all $x \in E$.

Proof. (a) The equality $S_{n}^{2}(w)=S_{n}(w)$ and orthogonality of $S_{n}$ are evident on a dense set of finite sums $w=\sum_{i} \alpha_{i} e_{1 i} \otimes \ldots \otimes e_{n i}$. The continuity of $S_{n}$ follows from the inequality

$$
\begin{aligned}
& \sup _{\|w\| \leq 1}\left\|S_{n}(w)\right\|=\sup _{\|w\| \leq 1}\left\|\frac{1}{n !} \sum_{\sigma \in \mathcal{G}_{n}} \sum_{i} \alpha_{i} e_{\sigma(1) i} \otimes \ldots \otimes e_{\sigma(n) i}\right\| \\
& \quad \leq \sup _{\|w\| \leq 1} \frac{1}{n !} \sum_{\sigma \in \mathcal{G}_{n}}\left[\sum_{i}\left|\alpha_{i}\right|^{2}\left\|e_{\sigma(1) i}\right\|^{2} \ldots\left\|e_{\sigma(n) i}\right\|^{2}\right]^{1 / 2}=\frac{1}{n !} \sup _{\|w\| \leq 1} n !\|w\|=1 .
\end{aligned}
$$

(b) Let $\phi \in E_{h}^{n \prime}$. From the Riesz Theorem it follows that there is a $w \in E_{h}^{n}$ such that $\phi(u)=(u \mid w)_{n}$. Put $P_{\phi}(x):=\left(x^{n} \mid w\right)_{n}$. It is clear that $P_{\phi}$ is an $n$-homogeneous polynomial on $E$. Since $\left|P_{\phi}(x)\right| \leq\|\phi\|\left\|x^{n}\right\|=$ $\|w\|\|x\|^{n}<\infty, P_{\phi}$ is bounded and so continuous. Denote by $\mathcal{P}_{h}\left({ }^{n} E\right)$ the subspace $\left\{P_{\phi}: \phi \in E_{h}^{n \prime}\right\}$ of $n$-homogeneous continuous polynomials with norm $\left\|P_{\phi}\right\|=\|\phi\|$. Evidently, $\mathcal{P}_{h}\left({ }^{n} E\right)$ is isometric to $E_{h}^{n \prime}$.

(c) By the polarization formula (see e.g. [7, p. 6]) and (b), for every $\phi \in E_{h}^{n \prime}$,

$$
\begin{aligned}
\phi\left(x_{1} \ldots x_{n}\right) & =A_{\phi}\left(x_{1}, \ldots, x_{n}\right)=\frac{1}{2^{n} n !} \sum_{\varepsilon_{i}= \pm 1} \varepsilon_{1} \ldots \varepsilon_{n} P_{\phi}\left(\sum_{j=1}^{n} \varepsilon_{j} x_{j}\right) \\
& =\frac{1}{2^{n} n !} \sum_{\varepsilon_{i}= \pm 1} \varepsilon_{1} \ldots \varepsilon_{n} \phi\left(\left(\sum_{j=1}^{n} \varepsilon_{j} x_{j}\right)^{n}\right)
\end{aligned}
$$

where $A_{\phi}$ is the symmetric $n$-linear form associated to $P_{\phi}$. Thus

$$
x_{1} \ldots x_{n}=\frac{1}{2^{n} n !} \sum_{\varepsilon_{i}= \pm 1} \varepsilon_{1} \ldots \varepsilon_{n}\left(\sum_{j=1}^{n} \varepsilon_{j} x_{j}\right)^{n} .
$$

Throughout, we will say that $E_{h}^{n}$ is the $n$-fold Hilbertian symmetric tensor product of $E$ and $\mathcal{P}_{h}\left({ }^{n} E\right)$ is the space of Hilbertian n-homogeneous polynomials on $E$. Define $u_{(j)}:=S_{n}\left(e_{j_{1}} \otimes \ldots \otimes e_{j_{n}}\right)$, where $(j)=\left(j_{1}, \ldots, j_{n}\right)$ is a multi-index. It is easy to see that $u_{(j)}=u_{(k)}$ if and only if there is a permutation $\sigma \in \mathcal{G}_{n}$ such that $\left(j_{1}, \ldots, j_{n}\right)=\left(k_{\sigma(1)}, \ldots, k_{\sigma(n)}\right)$. In this case we will say that the multi-indices $(j)$ and $(k)$ are equivalent. Denote by $\Gamma_{n}$ the set of equivalence classes of multi-indices, and by $[j]$ the element of $\Gamma_{n}$ containing $(j)$.

Lemma 1.1. The vectors $u_{[j]},[j] \in \Gamma_{n}$, form an orthogonal basis in $E_{h}^{n}$ and $1 \geq\left\|u_{[j]}\right\| \geq(1 / n !)^{1 / 2}$.

Proof. Clearly, $u_{[j]}$ and $u_{[k]}$ are orthogonal if $[j] \neq[k]$. Since the system $\left\{u_{[j]}\right\}$ is the image of a basis under the projection $S_{n}$, it is complete. Also 


$$
\begin{aligned}
& \left\|u_{[j]}\right\|=\left\|\frac{1}{n !} \sum_{\sigma} e_{j_{\sigma(1)}} \otimes \ldots \otimes e_{j_{\sigma(n)}}\right\| \\
& =\frac{1}{n !}\left\langle\sum_{\sigma} e_{j_{\sigma(1)}} \otimes \ldots \otimes e_{j_{\sigma(n)}} \mid e_{j_{\sigma(1)}} \otimes \ldots \otimes e_{j_{\sigma(n)}}\right\rangle^{1 / 2} \geq \frac{(n !)^{1 / 2}}{n !}=(1 / n !)^{1 / 2} .
\end{aligned}
$$

Corollary 1.1. Let $w \in E_{h}^{n}$ and $w=\sum_{i=1}^{\infty} \alpha_{i} e_{1 i} \ldots e_{n i}$. Then

$$
\left(\sum_{i=1}^{\infty}\left|\alpha_{i}\right|^{2}\right)^{1 / 2} \geq\|w\|^{2} \geq \frac{1}{\sqrt{n !}}\left(\sum_{i=1}^{\infty}\left|\alpha_{i}\right|^{2}\right)^{1 / 2} .
$$

Proof. Let $\widetilde{w}:=\sum_{i=1}^{\infty} \alpha_{i} e_{1 i} \otimes \ldots \otimes e_{n i}$. Then $w=S_{n}(\widetilde{w})$ and $\|\widetilde{w}\| \geq\|w\|$ since $S_{n}$ is an orthogonal projection. Since $\left\{e_{1 i} \ldots e_{n i} /\left\|e_{1 i} \ldots e_{n i}\right\|\right\}$ is an orthonormal basis in $E_{h}^{n}$, by Lemma 1.1 we have

$$
\|w\|^{2}=\sum_{i=1}^{\infty}\left|\alpha_{i}\right|^{2}\left\|e_{1 i} \ldots e_{n i}\right\|^{2} \geq \frac{1}{n !} \sum_{i=1}^{\infty}\left|\alpha_{i}\right|^{2} .
$$

It is clear that every polynomial of finite type is Hilbertian. Moreover, since every element $w \in E_{h}^{n}, w=\sum_{i=1}^{\infty} \alpha_{i} e_{1 i} \ldots e_{n i}$, is the limit of the sequence of the vectors $w_{k}=\sum_{i=1}^{k} \alpha_{i} e_{1 i} \ldots e_{n i}$, every Hilbertian polynomial can be approximated by polynomials of finite type in the norm of $\mathcal{P}_{h}\left({ }^{n} E\right)$. Since $\sup _{\|x\| \leq 1}|P(x)| \leq\|P\|$, every Hilbertian polynomial can be approximated by polynomials of finite type in the topology of uniform convergence on the unit ball of $E$. Thus if $P$ is a Hilbertian polynomial then $P$ is weakly continuous on bounded sets [3]. The converse is not true.

Proposition 1.2. There is a polynomial that is weakly continuous on bounded sets and not Hilbertian.

Proof. It is known that if $E^{\prime}$ has the approximation property then the space $\mathcal{P}_{\mathrm{w}}\left({ }^{n} E\right)$ of $n$-homogeneous polynomials weakly continuous on bounded sets is isomorphic to the injective symmetric tensor product $\bigotimes_{\varepsilon, \mathrm{s}}^{n} E^{\prime}$ of $E^{\prime}$ [8, p. 112]. On the other hand, $\mathcal{P}_{h}\left({ }^{n} E\right)=E_{h}^{n \prime} \subset \bigotimes_{\varepsilon, \mathrm{s}}^{n} E^{\prime}=\mathcal{P}_{\mathrm{w}}\left({ }^{n} E\right)$. This embedding is proper because $E_{h}^{n \prime}=E_{h}^{n}$ is a reflexive space but $\bigotimes_{\varepsilon, \mathrm{s}}^{n} E^{\prime}$ is not. More exactly, the adjoint space to the injective symmetric tensor product of the Hilbert space is the symmetric projective tensor product of the Hilbert space $\left[8\right.$, p. 112] which contains a copy of $\ell_{1}$. Note that since $\mathcal{P}_{h}\left({ }^{n} E\right)$ contains all polynomials of finite type and is a proper subspace of $\mathcal{P}_{\mathrm{w}}\left({ }^{n} E\right)$, the closure of $\mathcal{P}_{h}\left({ }^{n} E\right)$ in $\mathcal{P}\left({ }^{n} E\right)$ coincides with $\mathcal{P}_{\mathrm{w}}\left({ }^{n} E\right)$.

Let us recall that a polynomial $P$ is said to be integral if there exists a regular Borel measure $\mu$ of finite variation on $\left(\overline{\mathcal{B}}_{E^{\prime}}, \sigma\left(E^{\prime}, E\right)\right)$ such that

$$
P(x)=\int_{\overline{\mathcal{B}}_{E^{\prime}}} \phi(x)^{n} d \mu(\phi)
$$


for all $x \in E$, where $\phi \in \overline{\mathcal{B}}_{E^{\prime}}$. The Banach space of all $n$-homogeneous integral polynomials with the norm

$\|P\|_{\mathrm{I}}:=\inf \{\|\mu\|: \mu$ is a regular Borel measure of finite variation satisfying $(2)\}$

is denoted by $\mathcal{P}_{\mathrm{I}}\left({ }^{n} E\right)$. The concept of integral polynomials was introduced in $[6]$.

Proposition 1.3. The space $\mathcal{P}_{\mathrm{I}}\left({ }^{n} E\right)$ is a proper dense subspace of $\mathcal{P}_{h}\left({ }^{n} E\right)$.

Proof. Since $\mathcal{P}_{\mathrm{I}}\left({ }^{n} E\right)=\left(\bigotimes_{\varepsilon, \mathrm{s}}^{n} E\right)^{\prime}$ (see [6]) and $E_{h}^{n}$ is a dense proper subspace of $\bigotimes_{\varepsilon, \mathrm{s}}^{n} E$, it follows that $\mathcal{P}_{\mathrm{I}}\left({ }^{n} E\right)$ is a proper subspace of $E_{h}^{n \prime}=$ $\mathcal{P}_{h}\left({ }^{n} E\right)$. The density of $\mathcal{P}_{\mathrm{I}}\left({ }^{n} E\right)$ follows from the fact that $\mathcal{P}_{\mathrm{I}}\left({ }^{n} E\right)$ contains all polynomials which form an orthonormal basis in the Hilbert space $\mathcal{P}_{h}\left({ }^{n} E\right)$ and their linear span as well.

Therefore Propositions 1.2 and 1.3 imply that the space of Hilbertian polynomials lies strictly between the spaces of integral polynomials and polynomials weakly continuous on bounded sets.

It is known [10] that the space $\mathcal{P}\left({ }^{n} X\right)$ of all continuous polynomials (with sup-norm) on a Banach space $X$ with the approximation property is reflexive if and only if all polynomials from $\mathcal{P}\left({ }^{n} X\right)$ are weakly sequentially continuous, and this is equivalent to the fact that every $P \in \mathcal{P}\left({ }^{n} X\right)$ is norm attaining. The next theorem shows that for subspaces of $\mathcal{P}\left({ }^{n} X\right)$ the situation is different.

THEOREM 1.1. The set of norm attaining Hilbertian polynomials is not dense in $\mathcal{P}_{h}\left({ }^{n} E\right)$.

Proof. Let us show that a Hilbertian polynomial $P$ is norm attaining if and only if $P(x)=\left(x^{n} \mid v\right)_{n}$, where $v=y^{n}$ for some $y \in E$. Assume that $\|P\|=1$. Suppose that $P(y)=\|P\|$ for some $y \in E$ with $\|y\|=1$. Since

$$
\|P\|=\|v\|=(v \mid v)_{n}^{1 / 2}=(v \mid v)_{n}=\left(y^{n} \mid v\right)_{n}=1,
$$

we have $v=y^{n}$. In the general case, if $\|P\| \neq 0$, the polynomial

$$
P(x)=\|v\|\left(x^{n} \mid \frac{v}{\|v\|}\right)_{n}
$$

attains its norm if $v /\|v\|=y^{n}$ for some $y \in E$. Therefore if the set of norm attaining Hilbertian polynomials were dense in $\mathcal{P}_{h}\left({ }^{n} E\right)$ then the set $\left\{y^{n}: y \in E\right\}$ would be dense in $E_{h}^{n}$. But it is easy to check that the sum $e_{1} \otimes \ldots \otimes e_{1}+e_{2} \otimes \ldots \otimes e_{2}$ does not belong to the closure of this set.

2. Hilbert space of analytic functions. Let $\mathcal{H}(\mathcal{B})$ be the space of analytic functions on the unit ball $\mathcal{B}$. 
Definition. The Hardy-type space $H^{2}(\mathcal{B})$ is the subspace of $\mathcal{H}(\mathcal{B})$ consisting of all functions $f$ that can be expanded in the Taylor series

$$
f(x)=\sum_{n=0}^{\infty} f_{n}(x)
$$

with $f_{n}$ an $n$-homogeneous Hilbertian polynomial and

$$
\|f\|_{2}:=\left(\sum_{n=0}^{\infty}\left\|f_{n}\right\|^{2}\right)^{1 / 2}<\infty .
$$

Consider the $\ell_{2}$-direct sum of $E_{h}^{n}, \ell_{2}\left(E_{h}^{n}\right):=\bigoplus_{n=0}^{\infty} E_{h}^{n}$, where $E_{h}^{0}=\mathbb{C}$. The dual space $\ell_{2}^{\prime}\left(E_{h}^{n}\right)$ to $\ell_{2}\left(E_{h}^{n}\right)$ has a natural inner product $\langle\cdot \mid \cdot\rangle$.

THEOREM 2.1. The space $\ell_{2}^{\prime}\left(E_{h}^{n}\right)$ is isometrically isomorphic to the space $H^{2}(\mathcal{B})$.

Proof. Let $f \in \ell_{2}^{\prime}\left(E_{h}^{n}\right)$. By the isometric isomorphism $\ell_{2}^{\prime}\left(E_{h}^{n}\right) \simeq \ell_{2}\left(E_{h}^{n \prime}\right)$ there is a sequence $\left(\phi_{n}\right)$ of functionals such that

$$
\phi_{n} \in E_{h}^{n \prime} \quad \text { and } \quad f=\sum_{n=0}^{\infty} \phi_{n} .
$$

Let $\phi_{n}(x):=\left(x^{n} \mid w_{n}\right)_{n}$ for some $w_{n} \in E_{h}^{n}$ and $w_{0} \in \mathbb{C}$. We want to show that the operator

$$
f \mapsto f(x)=\langle\widehat{x} \mid f\rangle:=\sum_{n=0}^{\infty}\left(x^{n} \mid w_{n}\right)_{n}
$$

is the required isomorphism, where $x^{0}=1$ and $\phi_{0}=\left(x^{0} \mid w_{0}\right)_{0}:=w_{0}$.

For each $x=\zeta a \in \mathcal{B}, \zeta \in \mathbb{R},|\zeta|<1,\|a\|=1$, the vector

$$
\widehat{x}=\sum_{n=0}^{\infty} x^{n}
$$

belongs to the space $\ell_{2}\left(E_{h}^{n}\right)$, since its $\ell_{2}\left(E_{h}^{n}\right)$-norm is equal to

$$
\|\widehat{x}\|_{2} \equiv\left(\sum_{n=0}^{\infty}|\zeta|^{2 n}\right)^{1 / 2}=\frac{1}{\left(1-|\zeta|^{2}\right)^{1 / 2}} .
$$

From Schwarz's inequality it follows that

$$
|f(x)| \leq\|\widehat{x}\|_{2}\|f\|_{2}=\frac{\|f\|_{2}}{\left(1-|\zeta|^{2}\right)^{1 / 2}} .
$$

In other words, the function $f(x)$ is bounded in every ball $\{x=\zeta a:|\zeta| \leq$ 
$1-\varepsilon\}$, where $0<\varepsilon<1$. Moreover for $x+\zeta a \in \mathcal{B}$,

$$
\begin{aligned}
f(x+\zeta a) & =\langle\widehat{x+\zeta a} \mid f\rangle=\sum_{n=0}^{\infty} \sum_{k=0}^{n} \zeta^{k}\left(a^{k} x^{n-k} \mid w_{n}\right)_{n} \\
& =\sum_{k=0}^{\infty} \sum_{m=0}^{\infty} \zeta^{k}\left(a^{k} x^{m} \mid w_{k+m}\right)_{k+m} .
\end{aligned}
$$

Since $\left|\sum_{m=0}^{\infty}\left(a^{k} x^{m} \mid w_{k+m}\right)_{k+m}\right| \leq\|f\|_{2}\|\widehat{x}\|_{2}\|a\|^{k}$, the function $f(x)$ is Gateaux analytic and so [7] it is analytic in $\mathcal{B}$, i.e. $f(x) \in H^{2}(\mathcal{B})$.

Conversely, let $f(x) \in H^{2}(\mathcal{B})$. Then the coefficients $f_{n}$ of the Taylor series expansion (3) define a unique sequence of linear functionals $f_{n} \in E_{h}^{n \prime}$. So the sum $f=\sum_{n} f_{n}$ belongs to the space $\ell_{2}\left(E_{h}^{n \prime}\right)$.

Thus the required isomorphism is defined by the operator

$$
\ell_{2}^{*}\left(E_{h}^{n}\right) \ni f \mapsto f(x) \in H^{2}(\mathcal{B}), \quad \text { where } \quad f(x)=\langle\widehat{x} \mid f\rangle .
$$

Let $A$ be a selfadjoint operator on $E$ with domain $\mathcal{D}(A)$. Suppose that $-i A$ is the generator of a unitary group $\mathbb{R} \ni t \mapsto U_{t}$ of bounded operators.

Consider on $E \otimes \ldots \otimes E$ a group of operators

$$
\mathbb{R} \ni t \mapsto \overbrace{U_{t} \otimes \ldots \otimes U_{t}}^{n}
$$

such that

$$
\left(U_{t} \otimes \ldots \otimes U_{t}\right) w=\sum_{j} \alpha_{j} U_{t} x_{1 j} \otimes \ldots \otimes U_{t} x_{n j}
$$

for all $w=\sum_{j} \alpha_{j} x_{1 j} \otimes \ldots \otimes x_{n j} \in E \otimes \ldots \otimes E$. The generator of the group $U_{t} \otimes \ldots \otimes U_{t}$ has the representation

$$
-i\left(\sum_{j=1}^{n} A_{j}\right), \quad \text { where } \quad A_{j}=\overbrace{I \otimes \ldots \otimes I}^{j-1} \otimes A \otimes \overbrace{I \otimes \ldots \otimes I}^{n-j}
$$

and $I$ is the identity operator on $E$. Then we have

$$
\begin{gathered}
\left(U_{t} \otimes \ldots \otimes U_{t}\right) S_{n}=S_{n}\left(U_{t} \otimes \ldots \otimes U_{t}\right), \\
\widehat{A}_{n} x:=\left(\sum_{j=1}^{n} A_{j}\right) S_{n} x=S_{n}\left(\sum_{j=1}^{n} A_{j}\right) x
\end{gathered}
$$

for each $x$ from the domain of the generator $-i\left(\sum_{j} A_{j}\right)$. Set $A_{0}=0$.

Theorem 2.2. (a) The group of operators on $H^{2}(\mathcal{B})$ defined by

$$
\widehat{U}_{t} f(x)=f\left(U_{t} x\right), \quad t \in \mathbb{R}, x \in \mathcal{B}, f \in H^{2}(\mathcal{B}),
$$

is unitary.

(b) The generator of the group $\widehat{U}_{t}$ has the form $-i \widehat{A}$, where the operator $\widehat{A}=\mathcal{A}^{*}$ is adjoint with respect to the duality $\left\langle\ell_{2}\left(E_{h}^{n}\right) \mid H^{2}(\mathcal{B})\right\rangle$ to the diagonal 
matrix operator

$$
\mathcal{A}=\left(\begin{array}{ccccc}
0 & 0 & \ldots & 0 & \ldots \\
0 & A & \ldots & 0 & \ldots \\
\ldots \ldots \ldots \ldots & \ldots \ldots \ldots \ldots \ldots \\
0 & 0 & \ldots & S_{n} \sum_{j=1}^{n} A_{j} & \ldots \\
\ldots & \ldots & \ldots & \ldots & \ldots
\end{array}\right)
$$

defined on the subspace of finite sums of basis vectors in $\ell_{2}\left(E_{h}^{n}\right)$.

Proof. (a) Let $f=\sum f_{n}, g=\sum g_{n} \in H^{2}(\mathcal{B})$ and $f_{n}(x)=\left(x^{n} \mid u_{n}\right)_{n}$, $g_{n}(x)=\left(x^{n} \mid v_{n}\right)_{n}$, where

$$
u_{n}=\sum_{i=1}^{\infty} \alpha_{i} e_{1 i} \ldots e_{n i}, \quad v_{n}=\sum_{i=1}^{\infty} \beta_{i} e_{1 i} \ldots e_{n i} .
$$

Then, since $U_{t}$ is unitary,

$$
\begin{aligned}
\left\langle\widehat{U}_{t} f \mid \widehat{U}_{t} g\right\rangle & =\sum_{n=0}^{\infty}\left(\widehat{U}_{t} f_{n} \mid \widehat{U}_{t} g_{n}\right)_{n} \\
& =\sum_{n=0}^{\infty} \sum_{i=1}^{\infty} \alpha_{i} \beta_{i}\left(U_{t} e_{1 i} \mid U_{t} e_{1 i}\right) \ldots\left(U_{t} e_{n i} \mid U_{t} e_{n i}\right) \\
& =\sum_{n=0}^{\infty} \sum_{i=1}^{\infty} \alpha_{i} \beta_{i}\left(e_{1 i} \mid e_{1 i}\right) \ldots\left(U_{t} e_{n i} \mid U_{t} e_{n i}\right)=\langle f \mid g\rangle .
\end{aligned}
$$

(b) For every $n$ the space $E_{h}^{n}$ is an invariant subspace of $E \otimes \ldots \otimes E$ with respect to the action of the group $\widehat{U}_{t}$. Since $S_{n} \sum_{j} A_{j}$ is the generator of $\widehat{U}_{t}$ on $E_{h}^{n}$, the required assertion follows from (a).

Let $\sigma(A)$ be the spectrum of a selfadjoint operator $A$ and $A=\int_{\sigma(A)} \lambda d \mathcal{E}_{\lambda}$ its spectral decomposition, where the measure $\mathcal{E}_{\lambda}$ is concentrated on Borel subsets of $\sigma(A)$.

COROLlary 2.1. The following spectral decomposition holds:

$$
\widehat{A} f=\bigoplus_{n=0}^{\infty} \int_{\sigma(A) \times \ldots \times \sigma(A)}\left(\lambda_{1}+\ldots+\lambda_{n}\right) d \mathcal{E}_{\lambda_{1}} \otimes \ldots \otimes d \mathcal{E}_{\lambda_{n}} f_{n}
$$

for all $f=\sum_{n=0}^{\infty} f_{n} \in \mathcal{D}(\widehat{A})$, where

$$
\begin{aligned}
\mathcal{D}(\widehat{A}) & =\left\{f \in H^{2}(\mathcal{B}):\right. \\
& \left.\sum_{n=0}^{\infty} \int_{\sigma(A) \times \ldots \times \sigma(A)}\left(\left|\lambda_{1}\right|^{2}+\ldots+\left|\lambda_{n}\right|^{2}\right)\left(d \mathcal{E}_{\lambda_{1}} \otimes \ldots \otimes d \mathcal{E}_{\lambda_{n}} f_{n} \mid f_{n}\right)_{n}<\infty\right\} .
\end{aligned}
$$

Proof. Let us show first that $\sigma\left(\widehat{A}_{n}\right)=\left\{\lambda: \lambda=\lambda_{1}+\ldots+\lambda_{n}, \lambda_{k} \in \sigma(A)\right\}$. For a given operator $L$ with $\varrho(L) \neq \emptyset$, denote by $[L]$ the algebra of all rational 
functions of $L$. Since $[L]$ contains the resolvent of $L$, the operator $L-\lambda I$ is invertible if and only if it is invertible in $[L]$. So $\lambda \in \sigma(L)$ if and only if there exists a character (complex-valued homomorphism) $\phi$ on $[L]$ such that $\phi(L)=\lambda$. Indeed, suppose that $L-\lambda I$ is not invertible; then $L-\lambda I$ belongs to a maximal proper ideal in $[L]$. So there is a character $\phi$ such that $\phi(L-\lambda I)=0$ and $\phi(L)=\lambda$. Conversely, if $\phi(L)=\lambda$ then $L-\lambda I$ is not invertible and $\lambda \in \sigma(L)$.

Suppose that for some $k \geq 1, \sigma\left(\widehat{A}_{k}\right)=\left\{\lambda: \lambda=\sum_{i=1}^{k} \lambda_{i}, \lambda_{i} \in \sigma(A)\right\}$. (For $k=1$ this is evidently true.) Let $\theta$ be a character on $\left[\widehat{A}_{k}\right]$. For an arbitrary $B=\sum_{i=1}^{k} B_{i 1} \otimes \ldots \otimes B_{i, k+1} \in\left[\widehat{A}_{k+1}\right]$ put

$$
\bar{\theta}(B)=\sum_{i=1}^{k+1} \theta\left(B_{i 1} \otimes \ldots \otimes B_{i k}\right) I^{k} \otimes B_{i, k+1},
$$

where $I^{k}$ is the $k$ th tensor product of $I$. We claim that $\bar{\theta}$ is a homomorphism from $\left[\widehat{A}_{k}\right]$ to $I^{k} \times[A]$. Since $\left[\widehat{A}_{k+1}\right]$ is a one-generated commutative algebra, we need only show that $\bar{\theta}\left(\widehat{A}_{k+1}^{2}\right)=\left(\bar{\theta}\left(\widehat{A}_{k+1}\right)\right)^{2}$. It is easy to see that $\widehat{A}_{k+1}=$ $\widehat{A}_{k} \otimes I+I^{k} \otimes A$ and $\bar{\theta}\left(\widehat{A}_{k+1}\right)=I^{k} \otimes\left(A+\theta\left(\widehat{A}_{k}\right) I\right)$. Thus

$$
\begin{aligned}
& \bar{\theta}\left(\widehat{A}_{k+1}^{2}\right)=\theta\left(\widehat{A}_{k} \otimes I+I^{k} \otimes A\right)^{2} \\
& \quad=\theta\left(\widehat{A}_{k}^{2}\right) I^{k+1}+\theta\left(I^{k}\right) I^{k} \otimes A^{2}+2 \theta\left(\widehat{A}_{k}\right) I^{k} \otimes A \\
& \quad=I^{k} \otimes\left(A^{2}+\left(\theta\left(\widehat{A}_{k}\right)\right)^{2} I+2 A \theta\left(\widehat{A}_{k}\right)\right)=I^{k} \otimes\left(A+\theta\left(\widehat{A}_{k}\right) I\right)^{2}=\left(\bar{\theta}\left(\widehat{A}_{k+1}\right)\right)^{2} .
\end{aligned}
$$

Let now $\phi$ be a character on $\left[\widehat{A}_{k+1}\right]$ and $\phi\left(\widehat{A}_{k+1}\right)=\lambda$. For an arbitrary $\lambda_{0} \in \sigma\left(\widehat{A}_{k}\right)$ put $\lambda_{1}=\lambda-\lambda_{0}$. Then the operator

$$
\widehat{A}_{k+1}-\lambda I=\left(\widehat{A}_{k}-\lambda_{0} I\right) \otimes I+I \otimes \ldots \otimes I \otimes\left(A-\lambda_{1} I\right)
$$

is not invertible. Let $\theta_{0}$ be a character on $\left[\widehat{A}_{k}\right]$ such that $\theta_{0}\left(\widehat{A}_{k}\right)=\lambda_{0}$. Then

$$
\bar{\theta}\left(\left(\widehat{A}_{k}-\lambda_{0} I\right) \otimes I+I \otimes \ldots \otimes I \otimes\left(A-\lambda_{1} I\right)\right)=I^{k} A-I^{k} \lambda_{1}
$$

is a noninvertible operator. Thus $\lambda_{1} \in \sigma(A)$. So, from the induction assumption it follows that the spectrum of $\widehat{A}_{k+1}$ coincides with the $(k+1)$-fold sum of the spectrum of $A$.

The spectral measure $\mathcal{E}_{\lambda_{1}} \otimes \ldots \otimes \mathcal{E}_{\lambda_{n}}$ in $E_{h}^{n}$ and the measurable function $\sigma(A) \times \ldots \times \sigma(A) \ni\left(\lambda_{1}, \ldots, \lambda_{n}\right) \mapsto \lambda_{1}+\ldots+\lambda_{n}$ are induced by the selfadjoint operator

$$
\widehat{A}_{n}=\int_{\sigma(A) \times \ldots \times \sigma(A)}\left(\lambda_{1}+\ldots+\lambda_{n}\right) d \mathcal{E}_{\lambda_{1}} \otimes \ldots \otimes d \mathcal{E}_{\lambda_{n}}
$$

with domain 


$$
\left\{f_{n} \in E_{h}^{n}: \int_{\sigma(A) \times \ldots \times \sigma(A)}\left(\left|\lambda_{1}\right|^{2}+\ldots+\left|\lambda_{n}\right|^{2}\right)\left(d \mathcal{E}_{\lambda_{1}} \otimes \ldots \otimes d \mathcal{E}_{\lambda_{n}} f_{n} \mid f_{n}\right)_{n}<\infty\right\} .
$$

Now the required spectral decomposition of the operator $\widehat{A}$ follows from $[12$, Theorem 13.24].

\section{Wiener-type theorem for analytic functions on Hilbert spaces}

Lemma 3.1. The product of Hilbertian polynomials $P$ and $Q$ is a Hilbertian polynomial and $\|P\|\|Q\| \geq\|P Q\|$.

Proof. Let $P(x)=(x \mid u)_{n}$ and $Q(x)=(x \mid v)_{m}$ for some $u \in E_{h}^{n}$ and $v \in E_{h}^{m}$. Then $\|P Q\|=\left\|S_{n+m} u \otimes v\right\| \leq\|u \otimes v\|$. Let

$$
u=\sum_{i=1}^{\infty} \alpha_{i} e_{1 i} \ldots e_{n i}, \quad v=\sum_{i=1}^{\infty} \beta_{i} e_{1 i} \ldots e_{m i}
$$

Then

$$
\begin{aligned}
\|u \otimes v\|^{2} & =\sum_{i, j=1}^{\infty}\left|\alpha_{i}\right|^{2}\left|\beta_{j}\right|^{2}\left\|e_{1 i} \ldots e_{n i} \otimes e_{1 j} \ldots e_{m j}\right\|^{2} \\
& =\sum_{i, j=1}^{\infty}\left|\alpha_{i}\right|^{2}\left|\beta_{j}\right|^{2}\left\|e_{1 i} \ldots e_{n i}\right\|^{2}\left\|e_{1 j} \ldots e_{m j}\right\|^{2}=\|u\|^{2}\|v\|^{2}
\end{aligned}
$$

Denote by $W(\mathcal{B})$ the set of analytic functions on $\mathcal{B}$ such that $f(x)=$ $\sum_{k=1}^{\infty} P_{k}(x)$, where $P_{k} \in \mathcal{P}_{h}\left({ }^{k} E\right)$ and $\sum_{k=1}^{\infty}\left\|P_{k}\right\|<\infty$. Note that the functions from $W(\mathcal{B})$ are defined and bounded on $\mathcal{B}$, since

$$
\limsup _{k \rightarrow \infty} \frac{1}{\left\|P_{k}\right\|^{1 / k}} \geq 1
$$

(see e.g. [7, p. 166]). From Lemma 3.1 it follows that $W(\mathcal{B})$ is a Banach algebra with norm $\|f\|_{1}:=\sum_{k=1}^{\infty}\left\|P_{k}\right\|$.

The following lemma follows easily from the definition.

LEMMA 3.2. The algebra $W(\mathcal{B})$ is isomorphic to the convolution algebra $\ell_{1}\left(E_{h}^{n}\right)$ with $\left(u_{k}\right) *\left(v_{j}\right)=\left(w_{i}\right)$, where $w_{i}=\sum_{k=1}^{i} u_{k} v_{i-k}$ for $\left(u_{k}\right),\left(v_{k}\right) \in$ $\ell_{1}\left(E_{h}^{n}\right)$. Moreover $W(\mathcal{B})^{\prime}=\ell_{\infty}\left(E_{h}^{n}\right)$ as Banach spaces.

THEOREM 3.1. The set of maximal ideals of the algebra $W(\mathcal{B})$ is homeomorphic to the unit sphere $\mathcal{T}$ of $E$ in the Gelfand topology.

Proof. Let $\phi$ be a multiplicative linear functional on $W(\mathcal{B})$. By Lemma $3.2, \phi=\sum_{n=1}^{\infty} \phi_{n} \in\left(E_{h}^{n}\right)_{l_{\infty}}$, where $\phi_{n} \in E_{h}^{n}$ is the restriction of $\phi$ to $E_{h}^{n}$. Let $f \in W(\mathcal{B}), f(x)=\sum_{n=1}^{\infty} P_{n}(x)$. Then 


$$
\phi\left(f^{2}\right)=\sum_{k=1}^{\infty} \phi_{k} \sum_{n=1}^{k} P_{n} P_{k-n}
$$

On the other hand, from the multiplicativity of $\phi$ it follows that

$$
\phi\left(f^{2}\right)=(\phi(f))^{2}=\sum_{n=1}^{\infty} \phi_{n}\left(P_{n}\right) \sum_{m=1}^{\infty} \phi_{m}\left(P_{m}\right)=\sum_{k=1}^{\infty} \sum_{n=1}^{k} \phi_{n}\left(P_{n}\right) \phi_{k-n}\left(P_{k-n}\right) .
$$

Combining the last two formulas, we have $\phi_{n}\left(P_{1}^{n}\right)=\left(\phi_{1} P_{1}\right)^{n}$ for each $n$. Therefore $\phi_{n}^{n}=\phi_{1}^{n}$ on the dense subspace of finite type polynomials, and thus $\phi_{n}^{n}=\phi_{1}^{n}$ everywhere. Since the norm of a multiplicative functional is equal to one, $\left\|\phi_{1}\right\|=1$. Since every linear functional from $\in E^{\prime}$ is defined by some vector from $E$, we see that $\phi$ is the point evaluation functional at some point of the unit sphere. The Gelfand topology on this sphere coincides with the weakest topology for which all functions from $W(\mathcal{B})$ are continuous.

THEOREM 3.2. Let $f \in W(\mathcal{B})$ have no zeros on the unit sphere $\mathcal{T}$. Then $1 / f \in W(\mathcal{B})$.

Proof. Since $\mathcal{T}$ is the set of maximal ideals of $W(\mathcal{B}), f$ is invertible in $W(\mathcal{B})$ and so $f^{-1} \in W(\mathcal{B})$.

Acknowledgements. The second author was supported in part by Grant 01.07/00172 of Ukrainian Fund of Fundamental Investigations.

\section{References}

[1] R. Aron, B. Cole, and T. Gamelin, Spectra of algebras of analytic functions on a Banach space, J. Reine Angew. Math. 415 (1991), 51-93.

[2] - - - - Weak-star continuous analytic functions, Canad. J. Math. 47 (1995), 673-683.

[3] R. M. Aron, C. Hervés, and M. Valdivia, Weakly continuous mappings on Banach spaces, J. Funct. Anal. 52 (1983), 189-204.

[4] T. K. Carne, B. Cole, and T. W. Gamelin, A uniform algebra of analytic functions on a Banach space, Trans. Amer. Math. Soc. 314 (1989), 639-659.

[5] A. Defant and K. Floret, Tensor Norms and Operator Ideals, Math. Stud. 176, North-Holland, Amsterdam, 1993.

[6] S. Dineen, Holomorphy types on a Banach space, Studia Math. 34 (1977), 241-288.

[7] —, Complex Analysis on Locally Convex Spaces, Math. Stud. 57, North-Holland, Amsterdam, 1981.

[8] —, Complex Analysis on Infinite Dimensional Spaces, Springer, 1999.

[9] J. Diestel, H. Jarchow, and A. Tonge, Absolutely Summing Operators, Cambridge Univ. Press, 1995.

[10] J. A. Jaramillo and L. A. Moraes, Duality and reflexivity in spaces of polynomials, Arch. Math. (Basel) 74 (2000), 282-293. 
[11] M. Reed and B. Simon, Methods of Modern Mathematical Physics, Academic Press, New York, 1975.

[12] W. Rudin, Functional Analysis, McGraw-Hill, New York, 1973.

Institute of Mathematics

Cracow University of Technology

Warszawska 24

31-155 Kraków, Poland

E-mail: ovl@usk.pk.edu.pl
Institute for Applied Problems of Mechanics and Mathematics Ukrainian Academy of Sciences 3b, Naukova St.

79053, Lviv, Ukraine E-mail: sirand@mebm.lviv.ua

Reçu par la Rédaction le 23.4.2001

Révisé le 17.9.2002 